Supplemental Information

\title{
Visualizing Ligand-Mediated Bimetallic Nanocrystal Formation Pathways with in Situ Liquid Phase Transmission Electron Microscopy Synthesis
}

Mei Wang, ${ }^{1}$ Asher C. Leff, ${ }^{2,3}$ Yue Li, ${ }^{4}$ Taylor J. Woehl ${ }^{1, *}$

${ }^{1}$ Department of Chemical and Biomolecular Engineering, University of Maryland, College Park, MD 20742, USA

${ }^{2}$ Sensors and Electron Devices Directorate, Combat Capabilities Development Command, United States Army Research Laboratory, Adelphi, MD 20783, USA

${ }^{3}$ General Technical Services, LLC, Wall Township, New Jersey 07727

${ }^{4}$ Department of Chemistry \& Biochemistry, University of Maryland, College Park, MD 20742, USA 


\section{Outline}

1. Metal thiolate complex concentration for in situ LP-TEM synthesis

2. HRTEM images of nanocrystals formed by in situ LP-TEM and ex situ flask synthesis

3. HAADF-STEM and EDS of nanocrystals formed by in situ LP-TEM and ex situ flask synthesis

4. Ex situ flask synthesis from oxidized metal thiolate complex

5. Image analysis of particle size distribution, eccentricity, aggregated fraction

6. Radiolysis simulations of radical-ligand reactions

S7-S10 


\section{Metal thiolate complex concentration for in situ LP-TEM synthesis}

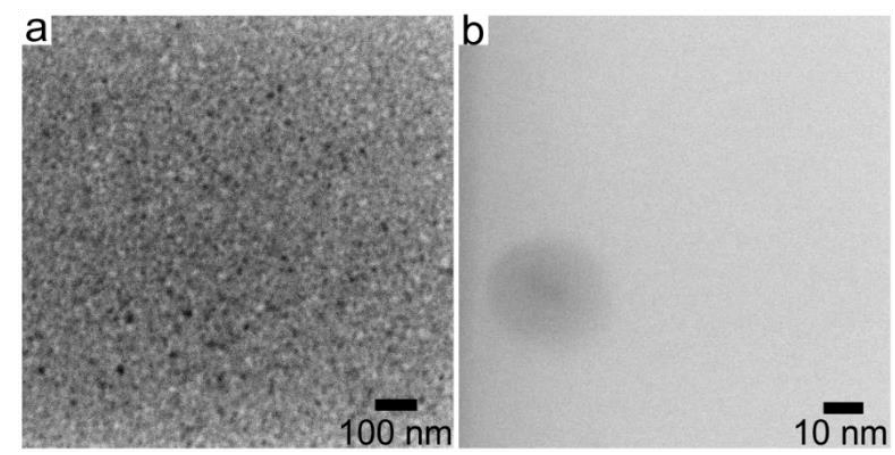

Figure S1. BF-STEM in situ LP-TEM images of nanocrystals formed from a metal thiolate precursor at (a) 7 times and (b) 70 times dilution compared to ex situ flask synthesis. (a) Synthesis time of $30 \mathrm{~s}$, beam current of $16 \mathrm{pA}$, and magnification of 250,000 (dose rate of $3.8 \mathrm{MGy} / \mathrm{s}$ ). (b) Synthesis time of $90 \mathrm{~s}$, beam current of $141 \mathrm{pA}$, and magnification of 1,200,000 (dose rate of 810 $\mathrm{MGy} / \mathrm{s})$.

2. HRTEM images of nanocrystals formed by in situ LP-TEM and ex situ flask synthesis

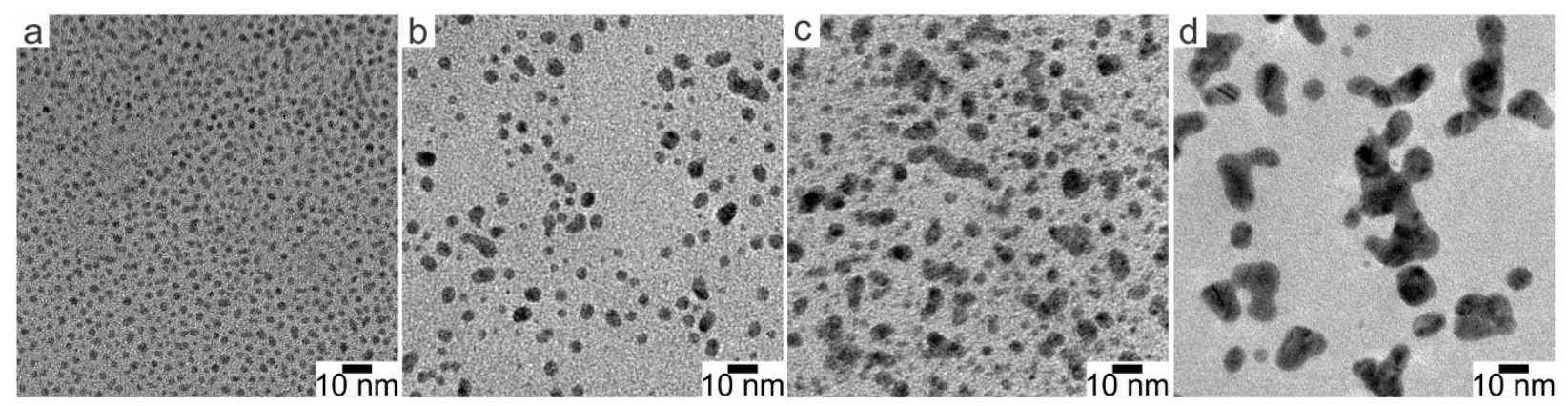

Figure S2. (a) HRTEM image of AuCu nanocrystals formed by ex situ flask synthesis. (b) In situ LP-TEM synthesis of $\mathrm{AuCu}$ nanocrystals from metal thiolate complexes at a magnification of 400,000 and beam current of $16 \mathrm{pA}$ (dose rate of 7.3 MGy/s). (c) In situ LP-TEM synthesis of Au nanocrystals formed from metal thiolate complexes at a magnification of 600,000 and beam current of $141 \mathrm{pA}$ (dose rate of $210 \mathrm{MGy} / \mathrm{s}$ ). (d) In situ LP-TEM synthesis of nanocrystals from a precursor solution with no ligand at a magnification of 400,000 and beam current of $141 \mathrm{pA}$ (dose rate of $65 \mathrm{MGy} / \mathrm{s})$. 


\section{HAADF-STEM and EDS of nanocrystals formed by in situ LP-TEM and ex situ flask}

synthesis
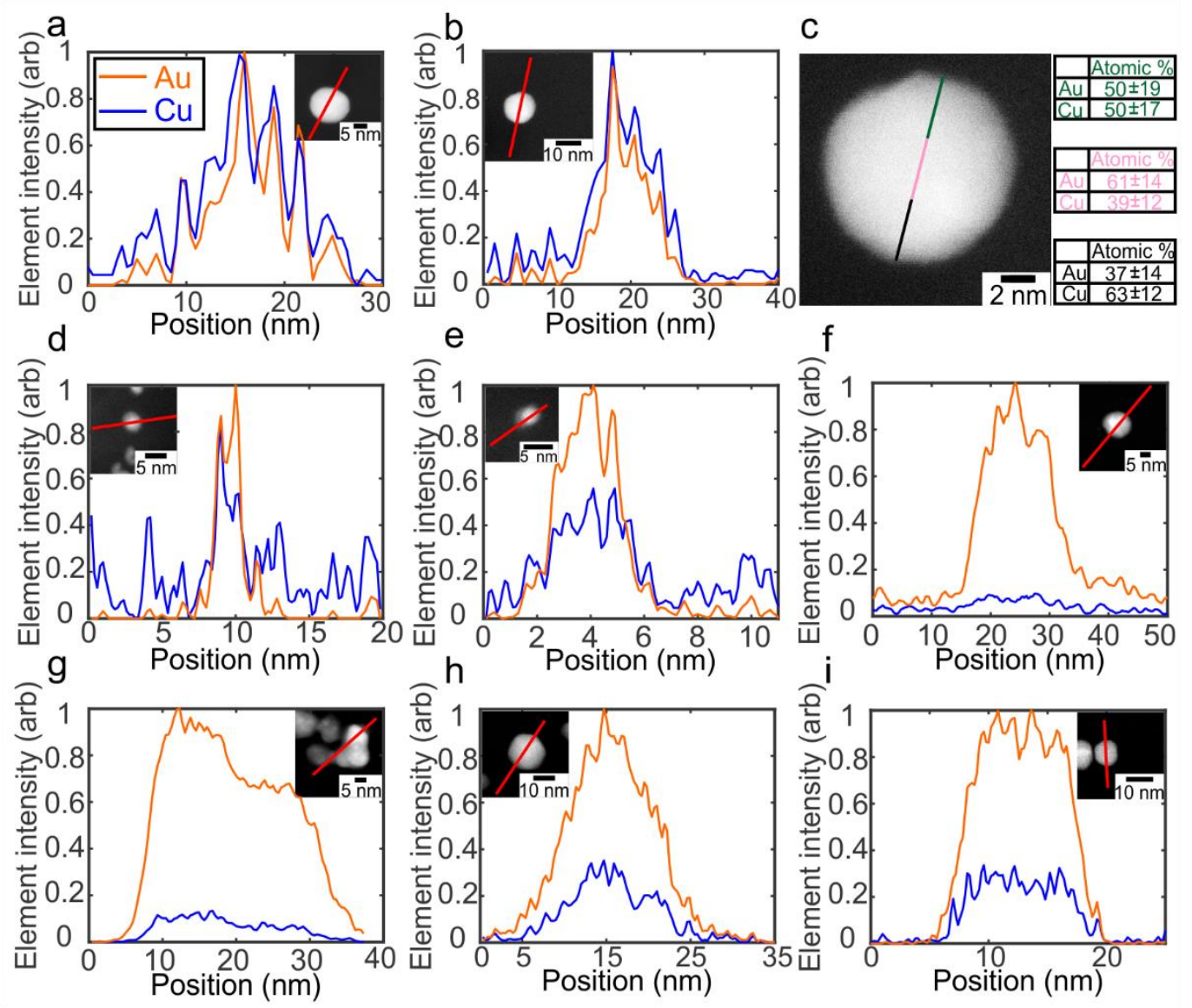

Figure S3. (a), (b) STEM-EDS line scans and (c) EDS composition at different positions of $\mathrm{AuCu}$ nanocrystals formed by in situ LP-TEM synthesis from metal thiolate complexes at low dose rate. (d), (e) STEM-EDS of AuCu nanocrystals formed by ex situ flask synthesis from metal thiolate complexes. (f) STEM-EDS of nanocrystals formed by in situ LP-TEM synthesis from metal thiolate complexes at high dose rate. (g) STEM-EDS of nanocrystal formed by in situ LP-TEM synthesis from a physical mixture of $\mathrm{HAuCl}_{4}$ and $\mathrm{Cu}\left(\mathrm{NO}_{3}\right)_{2}$. (h), (i) STEM-EDS of $\mathrm{AuCu}$ nanocrystals formed by ex situ flask synthesis from hydrogen peroxide oxidized metal thiolate complexes. 
Table SI. P-values for statistical analysis of alloy composition of nanocrystals synthesized with in situ LP-TEM synthesis, ex situ flask synthesis, and flask synthesis from $\mathrm{H}_{2} \mathrm{O}_{2}$ oxidized metal thiolate complexes. Each column represents a data pair. The Wilcoxon rank-sum test compared the medians of the two data sets while the Student's t-test compares the mean and variance. The outcome of the statistical test is shown in parentheses next to each p-value ( $R=$ reject null hypothesis, NR = do not reject null hypothesis); the null hypothesis was rejected if the p-value was $<0.01$.

\begin{tabular}{|c|c|c|c|}
\hline & $\begin{array}{c}\text { In situ LP-TEM/ } \\
\text { Ex situ flask }\end{array}$ & $\begin{array}{c}\text { Ex situ flask/ } \\
\text { Ex situ flask with } \\
\mathbf{H}_{\mathbf{2}} \mathbf{O}_{\mathbf{2}}\end{array}$ & $\begin{array}{c}\text { In situ LP-TEM/ } \\
E x \text { situ flask with } \\
\mathbf{H}_{2} \mathbf{O}_{2}\end{array}$ \\
\hline Wilcoxon rank-sum test & $0.19(\mathrm{NR})$ & $0.0001(\mathrm{R})$ & $0.0001(\mathrm{R})$ \\
\hline Student's t-test & $0.42(\mathrm{NR})$ & & \\
\hline
\end{tabular}

\section{Ex situ flask synthesis from oxidized metal thiolate complex}

To simulate the mixed oxidizing/reducing environment of in situ LP-TEM synthesis, $\mathrm{H}_{2} \mathrm{O}_{2}$ was added to the metal thiolate complex precursor solution at a final concentration of $1 \mathrm{mM}$ and allowed to react for 4 hours. The initial orange color of the metal thiolate complex solution disappeared after adding $\mathrm{H}_{2} \mathrm{O}_{2}$, indicating a change in metal oxidation state and/or metal coordinating ligands. MALDI-TOF MS of the oxidized solution showed the heteronuclear metal thiolate complexes were nearly entirely oxidized, resulting in a physical mixture of PEG-SH, PEGS-SPEG, and metal ions (not shown in the spectra) (Figure S4a). Nanocrystals formed from the oxidized solution were mostly spherical with increased size and polydispersity compared to those formed from the metal thiolate complex precursor (Figure S4b). 


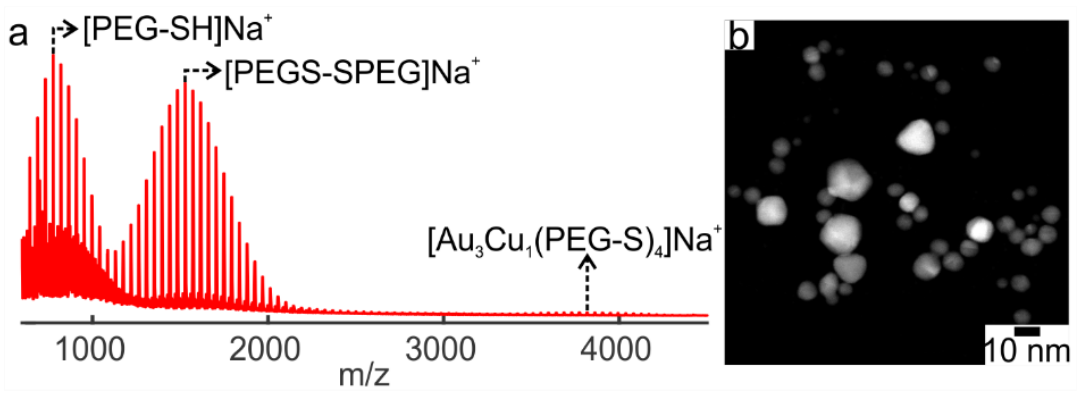

Figure S4. (a) MALDI-TOF-MS of PEG-SH mixed with $\mathrm{HAuCl}_{4}$ and $\mathrm{Cu}\left(\mathrm{NO}_{3}\right)_{2}$ at a molar ratio of 2:1:1 after reaction with $1 \mathrm{mM} \mathrm{H}_{2} \mathrm{O}_{2}$ for 4 hours. (b) HAADF-STEM of $\mathrm{AuCu}$ nanocrystals synthesized by ex situ flask synthesis from $\mathrm{H}_{2} \mathrm{O}_{2}$ oxidized metal thiolate complexes.

\section{Image analysis of particle size distribution, eccentricity, aggregated fraction heat map}

A MATLAB tracking algorithm was applied to the ex situ HRTEM images of nanocrystals to measure the particles size distribution (PSD), eccentricity, and aggregated fraction. Figure S5 shows an example of the particle tracking by the algorithm. Each particle in a TEM image was tracked and the particles' size and eccentricity were stored in the matrix (Figure S5a). Around 2000 particles were tracked from 3-5 different images at each experimental condition. Particles with eccentricity less than 0.75 were found by filtering out particles with high eccentricity from the particle tracking and counted as unaggregated particles (Figure S5b). The remaining particles were classified as aggregates. For each analyzed image/area, the average aggregated fraction was used to represent the aggregated fraction for each experimental condition.

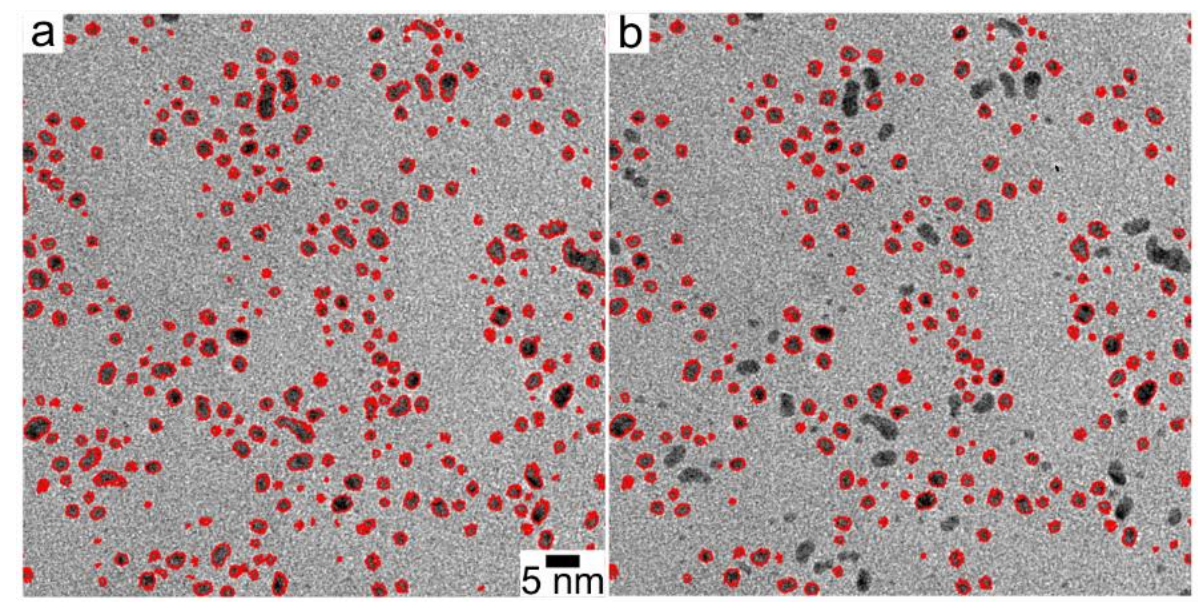

Figure S5. Example of particle tracking for (a) all particles and (b) unaggregated particles with eccentricity smaller than 0.75 (unaggregated outlined in red). 
To create the heat maps of eccentricity and aggregated fraction (Figure 4 in main text), the eccentricity and aggregated fraction of nanocrystals at each experimental condition were plotted as black points. 3D surfaces of eccentricity and aggregated fraction were fit to the scatter plots using triangulation-based cubic interpolation with a third degree polynomial.

\section{Radiolysis simulations of radical-ligand reactions}

The reaction kinetic model was implemented and solved in COMSOL using the Transport of Dilute Species Module. To calculate the concentrations of PEG-SH ligand and radical species associated with the radiation of PEG-SH ligands by the electron beam, we modified the reactiondiffusion model derived by N. Schneider and F.Ross ${ }^{1}$ by adding 17 additional chemical reactions to the original 79 primary reactions. The additional reactions with corresponding rate constants are summarized in Table SII. The rate constants of these reactions were estimated from literature values for pulse radiolysis measurements on small molecule sulfur compounds. ${ }^{2-7}$

Table SII. Additional chemical reactions associated with PEG-SH with significant rate constants.

\begin{tabular}{|c|c|}
\hline Chemical Reactions & Rate Constants $\left(M^{-1} S^{-1}\right)$,unless specified \\
\hline $\mathrm{PEG}-\mathrm{SH}+\mathrm{OH} \cdot \rightarrow \mathrm{PEG}-\mathrm{S} \cdot+\mathrm{H}_{2} \mathrm{O}$ & $1.0 \times 10^{10}$ \\
\hline $\mathrm{PEG}-\mathrm{SH}+\mathbf{e}_{\mathrm{aq}}^{-} \rightarrow \mathrm{PEG} \cdot+\mathrm{HS}^{-}$ & $8.7 \times 10^{9}$ \\
\hline $\mathrm{PEG}-\mathrm{SH}+\mathrm{H} \cdot \rightarrow \mathrm{PEG}-\mathrm{S} \cdot+\mathrm{H}_{2}$ & $1.0 \times 10^{9}$ \\
\hline 2PEG $-S \cdot \rightarrow$ PEGS - SPEG & $5.0 \times 10^{9}$ \\
\hline $\mathrm{PEG}-\mathrm{S} \cdot+\mathrm{O}_{2} \rightleftharpoons \mathrm{PEG}-\mathrm{SOO}$ & $k_{f}=2.0 \times 10^{9}, k_{b}=6.2 \times 10^{5} \mathrm{~s}^{-1}$ \\
\hline PEGS - SPEG + H $\cdot \rightarrow$ PEG $-\mathrm{S} \cdot+$ PEG - SH & $9.0 \times 10^{9}$ \\
\hline PEGS $-\mathrm{SPEG}+\mathrm{e}_{\mathrm{aq}}^{-} \rightarrow \mathrm{PEG}-\mathrm{S} \cdot+\mathrm{PEG}-\mathrm{S}^{-}$ & $4.5 \times 10^{9}$ \\
\hline 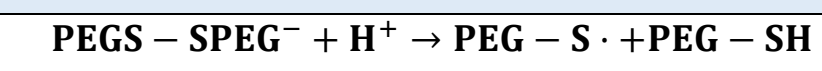 & $6.0 \times 10^{10}$ \\
\hline $\mathrm{PEG}-\mathrm{SH}+16 \mathrm{OH} \cdot \rightarrow \mathrm{PEG} \cdot-\mathrm{SH}+16 \mathrm{H}_{2} \mathrm{O}$ & $3.0 \times 10^{8}$ \\
\hline $\mathrm{PEG} \cdot-\mathrm{SH}+\mathrm{OH} \cdot \rightarrow \mathrm{PEG} \cdot-\mathrm{S} \cdot+\mathrm{H}_{2} \mathrm{O}$ & $1.0 \times 10^{10}$ \\
\hline PEG $\cdot-\mathbf{S H}+\mathbf{e}_{\mathrm{aq}}^{-} \rightarrow$ PEG $\cdots+\mathrm{HS}^{-}$ & $8.7 \times 10^{9}$ \\
\hline $\mathrm{PEG} \cdot-\mathrm{SH}+\mathrm{H} \cdot \rightarrow \mathrm{PEG} \cdot-\mathrm{S} \cdot+\mathrm{H}_{2}$ & $1.0 \times 10^{9}$ \\
\hline $\mathrm{PEG} \cdot-\mathrm{S} \cdot+\mathrm{O}_{2} \rightleftharpoons \mathrm{PEG} \cdot-\mathrm{SOO} \cdot$ & $k_{f}=2.0 \times 10^{9}, k_{b}=6.2 \times 10^{5} s^{-1}$ \\
\hline $2 \mathrm{PEG} \cdot-\mathrm{S} \cdot \rightarrow \mathrm{PEG} \cdot \mathrm{S}-\mathrm{S} \cdot \mathrm{PEG}$ & $5.0 \times 10^{9}$ \\
\hline $\mathrm{PEG} \cdot \mathrm{S}-\mathrm{S} \cdot \mathrm{PEG}+\mathrm{H} \cdot \rightarrow \mathrm{PEG} \cdot-\mathrm{S} \cdot+\mathrm{PEG} \cdot-\mathrm{SH}$ & $9.0 \times 10^{9}$ \\
\hline$P E G \cdot S-S \cdot P E G+e_{a q}^{-} \rightarrow P E G \cdot S-S \cdot P^{-} G^{-}$ & $4.5 \times 10^{9}$ \\
\hline
\end{tabular}


We calculated the time dependent concentration of species in 2D by numerically solving the reaction-diffusion equation:

$$
\frac{\partial C_{i}}{\partial t}=D_{i} \nabla^{2} C_{i}-\sum_{j} k_{i j} C_{i} C_{j}+\sum_{j, k \neq i} k_{j k} C_{j} C_{k}+R_{i}
$$

In this equation, the first term on the right-hand side represents the diffusion process of species $i$ with $D_{i}$ being the diffusion coefficient. The diffusion coefficient of species $i$ in solution was estimated with the Wilke-Chang Estimation Method, ${ }^{8}$ a modified Stokes-Einstein relation $D_{i}=$ $\frac{7.4 \times 10^{-8}\left(\varnothing M_{B}\right)^{1 / 2} T}{\eta_{B}\left(V_{i}\right)^{0.6}}$ where $M_{B}$ is the molecular weight of solvent, $\mathrm{T}$ is the temperature, $\eta_{B}$ is the viscosity of solvent, $V_{i}$ is the molar volume of species $i$, and $\varnothing=2.6$, a dimensionless factor when the solvent is water. The second and third terms on the right-hand side of equation S1 represent the destruction and production of species $i$ via chemical reactions. The last term represents the production of species $i$ by the electron beam. The geometry of this model was divided into two separate regions: the image region (Figure S6, red area) and the outer region (Figure S6, light blue area). In the image region, primary species were homogeneously generated by the electron beam and diffused into the outer region, where only recombination reactions occur.

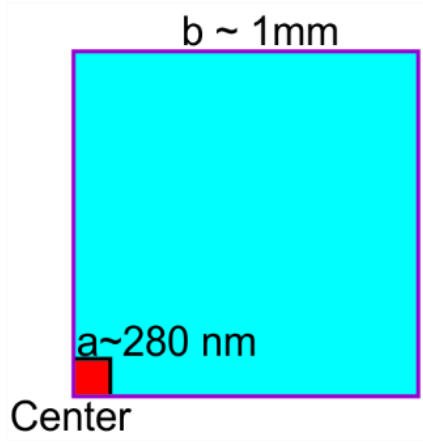

Figure S6. COMSOL simulation geometry.

The production rate of primary species by the electron beam in the image region was calculated by $R_{i}=\frac{\rho \Psi G_{i}}{F}$, where $\rho$ is the density of solvent, $\Psi$ is the dose rate, $G_{i}$ is the G-value of species $i, \mathrm{~F}$ is the faraday constant. $R_{i}=0$ in the outer region. The size of the image region was $280 \mathrm{~nm} \times 280 \mathrm{~nm}$, similar to the image region size during LP-TEM experiments. The size of the 
outer region was $1 \mathrm{~mm} \times 1 \mathrm{~mm}$, equal to half of the size of the fluid layer in the LP-TEM experiments. The initial concentrations of all the species was zero except $\mathrm{H}^{+}, \mathrm{OH}^{-}$and PEG-SH. No flux boundary conditions were applied to the edges of the outer region and the center due to symmetry (Figure S6. Purple lines). 56 differential equations in two regions associated with 28 chemical species were computed simultaneously to obtain the transient concentration profile of each species $i$ at different locations over 30 s. Figure S7a shows that the system reached steady state in less than $1 \mathrm{~s}$. To simplify the plots in Figure 5 in the main text and Figure S7, we combined the concentration of similar species with an alkyl radical on the PEG chain together with the species not having alkyl radicals on the PEG chain, [PEG-SH] $=[\mathrm{PEG}-\mathrm{SH}]+[\mathrm{PEG} \cdot-\mathrm{SH}]$, [PEG-S· $]$ $=[\mathrm{PEG}-\mathrm{S} \cdot]+[\mathrm{PEG} \cdot \mathrm{S} \cdot \mathrm{\cdot}, \quad[\mathrm{PEG} \cdot]=[\mathrm{PEG} \cdot]+[\mathrm{PEG} \cdot \cdot], \quad[\mathrm{PEGS}-\mathrm{SPEG}]=[\mathrm{PEGS}-\mathrm{SPEG}]+[\mathrm{PEG} \cdot \mathrm{S}-$ S.PEG]. The kinetics reached steady state in less than a second (Figure S7a). Figure S7b shows the steady state concentration of hydroxyl radicals and ligand damage products for a dose rate of 7.3 MGy/s and the concentration of each species was nearly uniform across the radiation area. The simulation showed that $26 \%$ of the initial PEG-SH ligands were converted to PEG $-\mathrm{S} \cdot, 55 \%$ to PEG $\cdot$, and $10 \%$ to PEGS-SPEG. The concentration of each species at the center of the image area was computed as a function of dose rate and showed that the PEG-SH and PEGS-SPEG concentrations decreased as a function of increasing dose rate, PEG-S. concentration increased slightly, and PEG · concentration remained nearly constant (Figure S7c). The $\mathrm{OH} \cdot$ concentration increased by nearly an order of magnitude when increasing dose rate from 7.3 MGy/s to 210 $\mathrm{MGy} / \mathrm{s}$.
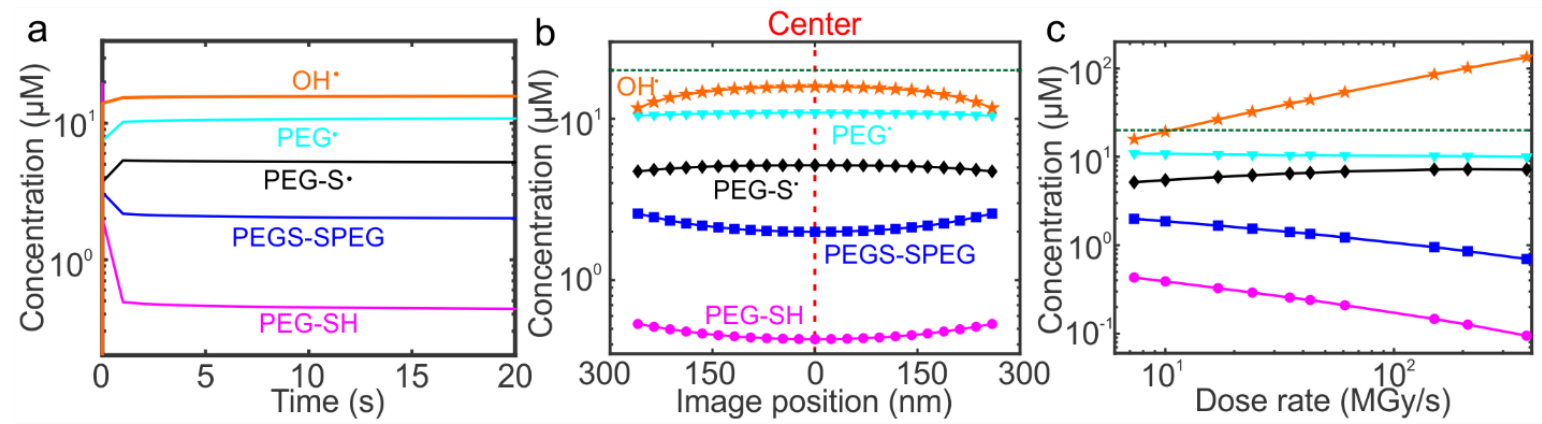

Figure S7. (a) Concentration of radical and molecular species at the center of the image area as a function of time for a magnification of 400,000 and beam current of $16 \mathrm{pA}$ (dose rate of 7.3 MGy/s). (b) Steady state concentration of radical and molecular species as a function of position within the 
LP-TEM image area for a magnification of 400,000, beam current of $16 \mathrm{pA}$ (dose rate of 7.3 MGy/s). (c) Steady state concentration of each species at the center of the LP-TEM image area as a function of dose rate. The horizontal dashed green lines in (b) and (c) show the $20 \mu \mathrm{M}$ initial concentration of PEG-SH.

\section{References}

1. Schneider, N. M.; Norton, M. M.; Mendel, B. J.; Grogan, J. M.; Ross, F. M.; Bau, H. H., Electron-Water Interactions and Implications for Liquid Cell Electron Microscopy. J. Phys. Chem. C 2014, 118, 22373-22382.

2. Hoffman, M. Z.; Hayon, E., Pulse Radiolysis Study of Sulfhydryl Compounds in Aqueous Solution. J. Phys. Chem. 1973, 77, 990-996.

3. Neta, P.; Schuler, R. H., Rate Constants for Reaction of Hydrogen Atoms with Compounds of Biochemical Interest. Radiat. Res. 1971, 47, 612-627.

4. Hoffman, M. Z.; Hayon, E., One-Electron Reduction of the Disulfide Linkage in Aqueous Solution. Formation, Protonation, and Decay Kinetics of the RSSR-Radical. J. Am. Chem. Soc. 1972, 94, 7950-7957.

5. $\quad$ Tung, T.-L.; Kuntz, R. R., The Reactions of Hydrogen Atoms in Aqueous Solutions: Thiols. Radiat. Res. 1973, 55, 10-17.

6. Matheson, M. S.; Mamou, A.; Silverman, J.; Rabani, J., Reaction of Hydroxyl Radicals with Polyethylene Oxide in Aqueous Solution. J. Phys. Chem. 1973, 77, 2420-2424.

7. Tamba, M.; Simone, G.; Quintiliani, M., Interactions of Thiyl Free Radicals with Oxygen: A Pulse Radiolysis Study. Int. J. Radiat. Biol. Relat. Stud. Phys., Chem. Med. 1986, 50, 595-600.

8. $\quad$ Poling, B. E.; Prausnitz, J. M.; O'Connell, J. P., The Properties of Gases and Liquids. 5th ed. ed.; McGraw-Hill: New York, 2001. 